\title{
Identification of potential key genes associated with ovarian clear cell carcinoma
}

\section{Youzheng Xu \\ Keng Shen}

Department of Obstetrics and Gynecology, Peking Union Medical College Hospital, Chinese Academy of Medical Sciences and Peking Union Medical College, Eastern District, Beijing 100730, China
This article was published in the following Dove Press journal: Cancer Management and Research

Background: Ovarian cancer is the major cause wide. Ovarian clear cell carcinoma (OCCC) is nsider a distinct mstopathologic subtype with worse prognosis and resistance to con tion anerar

Materials and methods: We analy five micro ay asets derived from the Gene Expression Omnibus database. GE $R$ to was used to reen out differentially expressed genes (DEGs) between OCCC tumor and no al ovary tissue. Gene Ontology and Kyoto Encyclopedia of Genes and cnomes pathway ent anentysis were performed using the g:Profiler database and Cy scape. Based $n$ Search Tool for the Retrieval of Interacting Genes, we performed protein-pro $n$ interaction $\mathrm{PI}$ ) network analysis on the DEGs. Real-time PCR (RT-PCR) and Western blott. in frozer amples of normal ovary and OCCC were performed to verify the ex differenow a nenes in OCC patients.

Results: Thirty u egula GGs and 13 downregulated DEGs were identified by cross referencing $\mathrm{C}_{\mathrm{i}}$ were $\mathrm{c}$ as hub genes with high connectivity degree via PPI network analysis, inc ang to upregu ted and four downregulated. RT-PCR and Western blotting results showed onificant znression ifference of the two upregulated genes, SPP1 and EPCAM, between tù a normariosues.

Conc rion: Our research suggests that SPP1 and EPCAM are overexpressed in OCCC compare ith normal ovary tissue. Clinical study of large sample is required to evaluate he value of SPP1 and EPCAM in the precision treatment and prognostic influence on OCCC in future.

Keywords: ovarian clear cell carcinoma, differentially expressed genes, SPP1, EPCAM

\section{Introduction}

Ovarian cancer accounts for about $4 \%$ of worldwide cancer incidence and mortality among women. As the seventh most common cancer and the eighth leading cause of cancer-related death in 2012 with 238,700 cases and 151,900 deaths, ${ }^{1}$ ovarian cancer has nonspecific symptoms, causing more than $60 \%$ of cases to be diagnosed at late stage, with a 5 -year survival rate of $30 \%-40 \%$ in most countries. ${ }^{2}$ Ovarian clear cell carcinoma (OCCC) is considered a rather intriguing subtype among ovarian cancers due to its distinct histopathologic subtype, worse prognosis, and resistance to conventional platinum-based chemotherapy. Studies showed that OCCC has higher prevalence rate in East Asia (15\%-25\%) than in North America and Europe $(1 \%-12 \%)$ due to race difference. ${ }^{3}$ OCCC occupies less than $5 \%$ of all ovarian cancers. ${ }^{4}$ An increased body mass index $>30$ and endometriosis are associated with this histological subtype on the basis of several studies with an OR of 2.2-2.3. ${ }^{5}$
Correspondence: Keng Shen Department of Obstetrics and Gynecology, Peking Union Medica College Hospital, Chinese Academy of Medical Sciences and Peking Union Medical College, No. I Shuai Fu Yuan, Eastern District, Beijing 100730, China Tel $+86 I 78 I 025902 I$ Email shenkeng@pumch.cn 
High levels of vascular endothelial factor (vascular endothelial growth factor) expression were revealed in OCCC, correlating with shorter survival. Upregulation of IL-6/STAT-3/hypoxia-inducible factor signaling can also be found in OCCC, which is fundamental in hypoxia-induced angiogenesis. ${ }^{6}$ HER 2 is overexpressed in $14 \%$ of OCCCs, ${ }^{7}$ suggesting a further potential therapeutic agent. Regarding somatic mutations of OCCC, mutations of PIK3CA (32\%-33\%), ARIDIA (46\%), KRAS, and BRAF are frequently presented. ${ }^{8-10}$

Though rarely seen, reliable genetic diagnosis and target therapy for the precise treatment of OCCC patients are needed as its poor prognosis and resistance in chemotherapy. Both clinical approaches and genomic approaches are necessary in this quest. ${ }^{11}$ However, low incidence of OCCC and small number of samples bring obstacles in clinical trial, experimental research, and genomic analysis.

In this study, bioinformatical methods were applied to detect the differentially expressed genes (DEGs) between OCCC and normal human ovary tissue on gene expression profiling data downloaded from the Gene Expression Omnibus (GEO) database. Gene Ontology (GO) function and Kyoto Encyclopedia of Genes and Genomes (KEGG) pathway enrichment analysis and protein-protein interactio (PPI) network analysis were performed to detect novel ind cators of OCCC patients and endeavor to provide potential therapeutic targets for this unique disease.

\section{Materials and methods}

Microarray data download and pro essing The gene expression profiles GS 008 , GSE 450 , GSE18520, GSE54388, and SE63885 re downloaded from GEO database (http www ncbi.nlm. gov/geo/). GSE6008 was based GPL [HG-U133A] Affymetrix Human Genome U1. array $\mathrm{p}^{1}$ iorm. GSE29450, GSE18520, G 54 . 8, an GSP 885 were based on GPL570 [H U133 Lus_2] Alymetrix Human Genome U133 plus 2 a ay pratrum. GSE63885 contains no normal ovary ue samples, while GSE18520 and GSE54388 contain OCCC tissue samples; these three datasets were merged into one named "Dataset C" before further analysis. The original CDF files of the platform and CEL files of the arrays were downloaded from GEO website and Gene Chip Robust Multichip Average was used for normalization, which can adjust the background intensity and normalize the probe intensity of Affymetrix data in the merging process. The data after normalization are exported as Dataset $\mathrm{C}$ and analyzed. All datasets were renormalized at the probe level before analysis. All of the data were freely available online.

\section{Tumor and normal ovary samples}

Four tumor samples came from four OCCC patients treated at the Peking Union Medical College Hospital, Beijing, China. All patients had been treated with initial cytoreductive surgery followed by platinum-based chemotherapy. Four normal ovary tissue samples came from four benign disease patients surgically treated at the Peking Union Medical College Hospital. All samples were stored in liquid nitrogen tank after was approved by the ethics con ittee of Pe $\mathrm{g}$ Union Medical College Hospital. A 1 pa ts provid w written informed consent before study. 1 wa conducted in accordance with th seclarg on of Huki. All data were de-identified

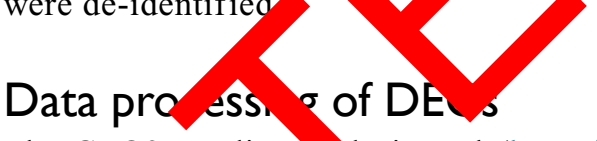

The GEO2R online a lysis tool (https://www.ncbi.nlm. nih.g geo/geo2r) was ined to detect the DEGs between OC $C$ and norm samples, and the adjusted $P$-value and $\mid \log 2$ C $\mid$ were cal lated. Genes that met the cutoff criteria, adjuste $<0$ of $n d|\log 2 \mathrm{FC}|>2.0$, were considered as DEGs. D. dataset owns unique DEGs. Venn diagram tool (online) ap://ownfogp.cnb.csic.es/tools/venny) was used to analyze overlapping components.

\section{GO and KEGG pathway analysis of DEGs}

$\mathrm{GO}$ analysis divides gene functions into biological process (BP), molecular function (MF), and cellular component (CC). KEGG analyzes genomes, biological pathways, diseases, chemical substances, and drugs on the DEGs. $\mathrm{g}$ :Profiler database (https://biit.cs.ut.ee/gprofiler/) and Cytoscape platform were used to identify the pathways and functional annotation of found genes and visualization of results. $P<0.05$ and gene counts $>10$ were considered statistically significant.

\section{PPI network and module analysis}

To evaluate the interactive relationships among DEGs, we mapped the DEGs to the Search Tool for the Retrieval of Interacting Genes (STRING) database (http://string-db.org/) with a combined score $>0.4$. PPI networks were constructed using the Cytoscape software. Nodes with higher degree of connectivity tend to be more essential in the functional network. The top six genes with degree of connectivity $>10$ were identified as hub genes. 


\section{Real-time PCR}

Total RNA was isolated from tissues with TRIzol reagent (Invitrogen) according to the manufacturer's instructions. Complementary DNA was synthesized by reverse transcription GoScript ${ }^{\mathrm{TM}}$ Reverse Transcription System (Promega, Madison, WS, USA). Quantitative real-time PCR (qPCR) analysis used the GoTaq ${ }^{\circledR}$ qPCR Master Mix (Promega). The primer sets are shown in Table S1. The PCR amplification was performed for 40 cycles of $95^{\circ} \mathrm{C}$ for 5 seconds and $60^{\circ} \mathrm{C}$ for 30 seconds, and melting curve reaction was performed at the end. All data analyses were operated using the 7500 Fast RealTime PCR Systems (Applied Biosystems). The $\Delta \Delta C t$ method was used to assess the relative expression of different genes.

\section{Western blotting analysis}

Tissues were collected and lysed in RIPA (Thermo Fisher Scientific, Waltham, MA, USA) buffer supplemented with phenyl-methane-sulfonyl fluoride (Boster Biology, Pleasanton, CA, USA). The concentration of protein samples was detected using a BCA Protein Assay Kit (Beyotime Institute of Biotechnology). Equal amounts of lysates were separated by $10 \%$ SDS-PAGE gels and transferred to polyvinylidene difluoride membranes. Membranes were blocked for 1 hour in BSA blocking buffer (Solarbio Life Sciences, Beijing, China) and probed with primary antibodies (at a dilution of 1:1, at $4^{\circ} \mathrm{C}$ for 12 hours (rabbit monoclonal to $W T 1$, resea resource identifier (RRID): ab89901; rabbit SPP1, RRID: ab8448; rabbit polyclonal o $D C A$ RRID ab175404; rabbit polyclonal to $E P C$

\section{CAM, TRID}

rabbit monoclonal to $A L D H 1 A 1$ RID: a 492; rabbit polyclonal to Gata6, RRID: ab22 and rabbit p clonal to beta actin, RRID: ab8227). Al were $\mathrm{p}_{\mathrm{c}}$ hased from Abcam Corporation. Then they vere incubated ith the specific horse radish peroxid (HRP conjugated secondary antibody (goat antirabbit $\mathrm{G}, \mathrm{P}$ [ab6721] from Abcam) for 2 hours before $\quad$ ing the the $L$ kit (Merck Millipore, Kenilwort NJ, Us A). Data alysis was performed using
ImageJ software to evaluate the expression levels of proteins. Statistical analysis was performed using SPSS 17.0 software (IBM Corp., Armonk, NY, USA) and with GraphPad Prism, version 5 (GraphPad Software). Statistically significant differences $(P<0.01)$ were determined by Student's $t$-test or ANOVA in the RT-PCR test and Western blotting analysis, presented as mean $\pm \mathrm{SD}$.

\section{Results \\ Identification of DEGs}

Five microarray datasets (GSE6008, GSE29450, GSE18520, GSE54388, and GSE63885) and mber of tumor and normal ovary tissue sam or are sho in Table 1. GSE63885 contains no norm ovary tis e samples. GSE18520 and GSE54? contain OC tissue samples. To accomplish comp son bo en normal ovary tissue and tumor thes hre catasets yere merged into one named "Data C". The EO2R Mline analysis tool was used to id at) DEGs sepa y with the cutoff criteria, adjusted $P<0.05$ a $\quad|\log 2 \mathrm{FC}|>2.0$ (Table 1 ), to compare OC comples with mal ovary samples. DEGs expresIn heat map and volcano plots are shown in Figures 1 a 2. Venn an ysis was performed to get the intersection of DEG $p$ files (Figure 3). Finally, 43 DEGs were significantry uifferentially expressed among all three groups, 1h 30 were significantly upregulated genes and 13 were downregulated genes.

GO function and KEGG pathway enrichment analysis of DEGs g: Profiler were used to analyze GO function and KEGG pathway enrichment for DEGs (Table 2). The enriched GO terms were divided into $\mathrm{CC}, \mathrm{BP}$, and MF ontologies. DEGs were mainly enriched in BPs, including tissue development, epithelium development, epithelial cell differentiation, tube development, organ development, and morphogenesis. MF analysis showed that the DEGs were significantly enriched in protein binding. For cell component, the DEGs were enriched in extracellular region, organelle,

Table I Three analyzed datasets and corresponding DEGs

\begin{tabular}{|c|c|c|c|c|c|c|}
\hline \multicolumn{2}{|c|}{ Dataset ID } & OCCC & Normal & Platform & Upregulated genes & $\begin{array}{l}\text { Downregulated } \\
\text { genes }\end{array}$ \\
\hline \multicolumn{2}{|c|}{ GSE6008 } & 8 & 4 & HG-UI33A & 70 & 53 \\
\hline \multicolumn{2}{|c|}{ GSE29450 } & 10 & 10 & HG-UI33_Plus_2 & 805 & 599 \\
\hline \multirow[t]{2}{*}{ C } & GSEI8520 & \multirow[t]{2}{*}{9} & \multirow[t]{2}{*}{16} & \multirow[t]{2}{*}{ HG-UI33_Plus_2 } & \multirow[t]{2}{*}{580} & \multirow[t]{2}{*}{231} \\
\hline & $\begin{array}{l}\text { GSE54388 } \\
\text { GSE63885 }\end{array}$ & & & & & \\
\hline
\end{tabular}

Abbreviations: DEGs, differentially expressed genes; OCCC, ovarian clear cell carcinoma. 
A

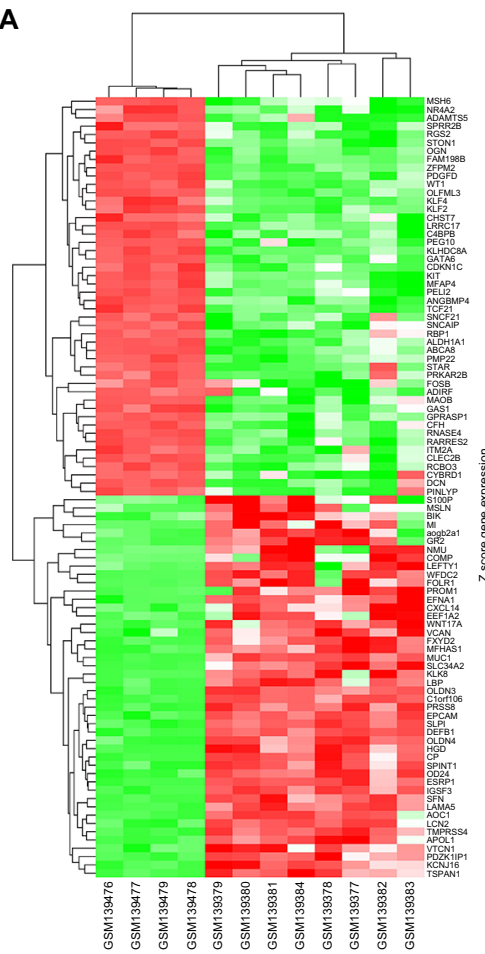

B

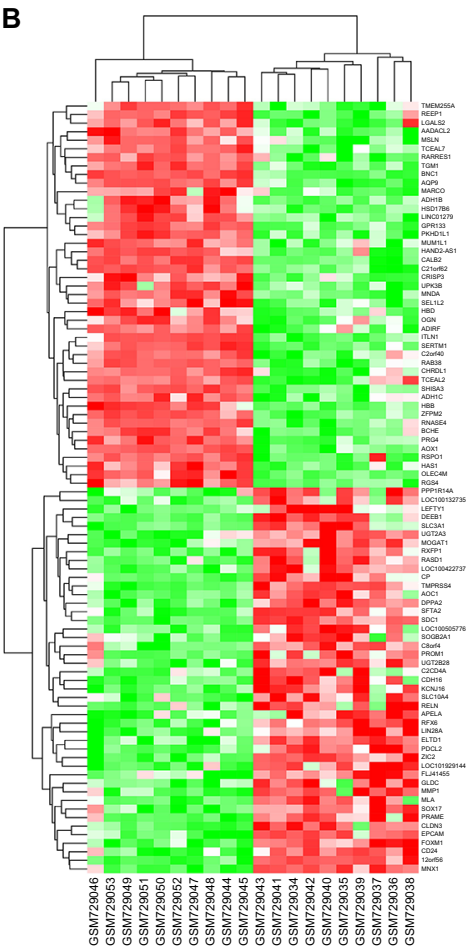

C

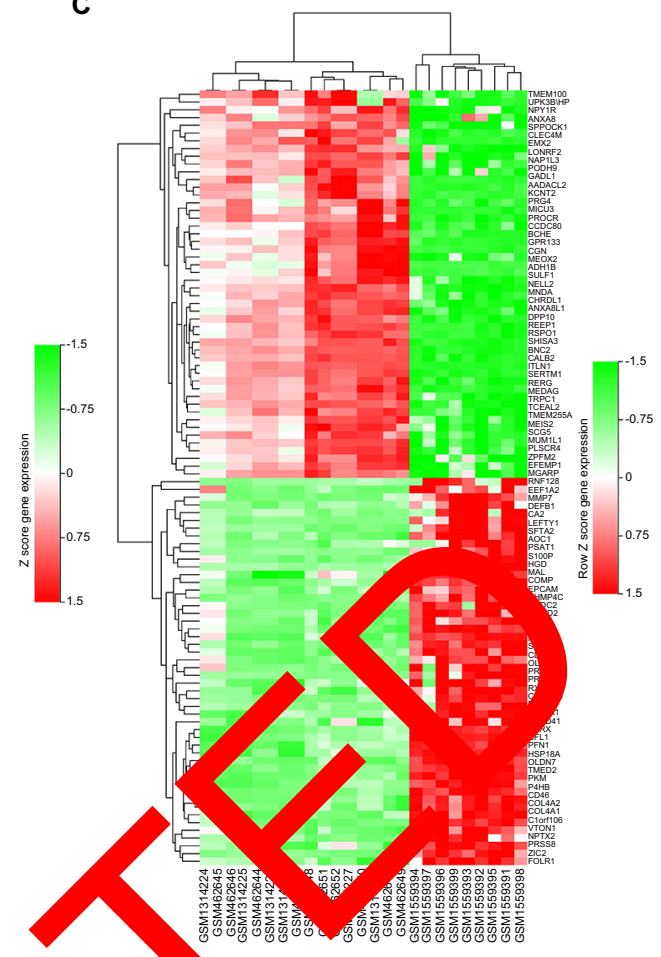

Figure I Heat maps of the differentially expressed genes of (A) GSE6008, (B) GSE29450, and (C) Datam Note: Red: upregulation; green: downregulation.

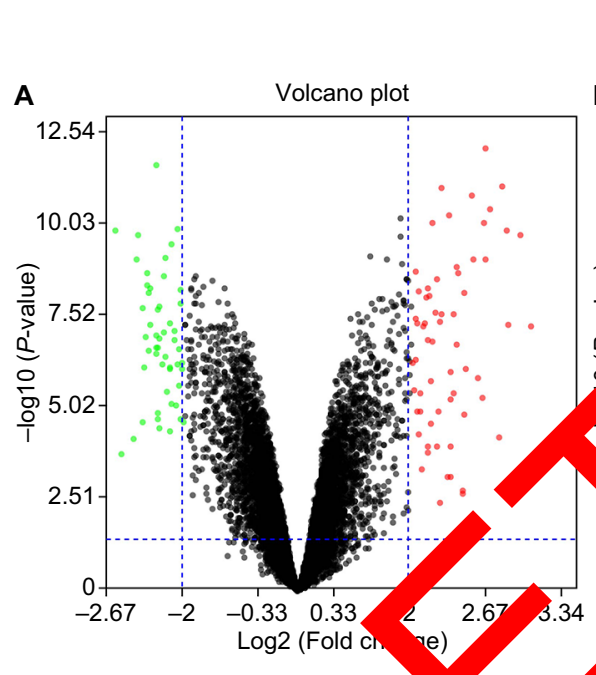

Figure 2 Volcano plots of Note: Red: upregulatio

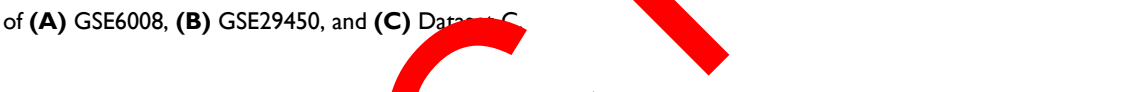

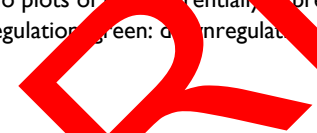

and space. The re s of KEGG pathway analysis showed that DEGs were mas enriched in extracellular matrix (ECM)-receptor interaction pathway and TGF-beta signaling pathway.

\section{PPI network analysis}

To explore the potential relationship between the aberrantly expressed genes, we performed a PPI network analysis with the online software STRING (score $>0.4$ ). Results were visualized by Cytoscape, as presented in Figure 4. The top six genes evaluated by connectivity degree in the PPI network were identified (Table 3). Two of these hub genes (SPP1, EPCAM) are upregulated in OCCC.

\section{RT-PCR and western blotting analysis}

We used four OCCC tumor tissue samples and four normal ovary tissue samples to evaluate the expression level of the six hub genes by RT-PCR and Western blotting analysis. As is shown in Figure 5A, B, mRNA and protein levels of both $S P P 1$ and $E P C A M$ were significantly upregulated in 


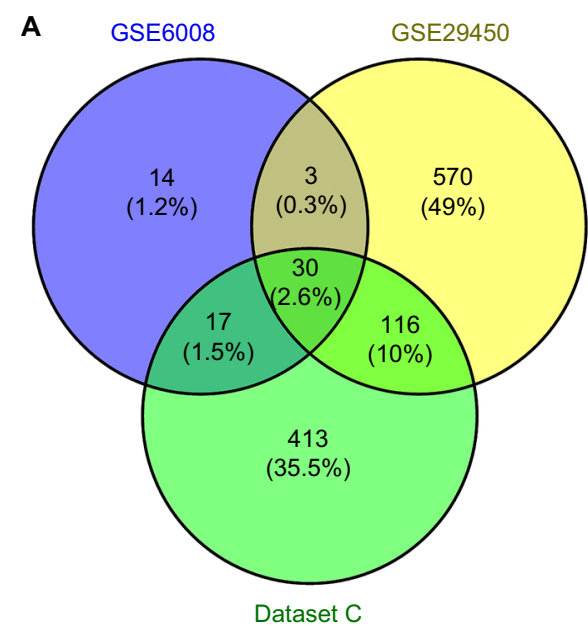

Figure 3 Venn diagram of DEGs common to all three datasets. Notes: (A) Upregulated genes. (B) Downregulated genes. Abbreviation: DEGs, differentially expressed genes.

carcinoma than normal tissues $(P<0.01)$. However, the other four downregulated genes showed no significant difference between two groups.

\section{Discussion}

OCCC is a distinct histopathologic subtype of ovarian cancer. Other than the common characteristics of nonspecific symptoms and low survival rate of ovarian cancer, OCCC sho worse prognosis and resistance to conventional platinum based chemotherapy, resulting in substantial obstroles for cancer treatment. Although OCCC only occy res les than $5 \%$ of all ovarian cancers, efforts have b n devo into the clinical and experimental research o $\mathrm{OQ}$ which reliable genetic diagnosis and targ herapy rem essential but unclear. Both clinical a gen ic approac.es are necessary in this quest. Ho ver, low inc nce of OCCC and small number of say es brinc obstacles inclinical trial, experimental research, ger mic analysis.

In the present ondy, fir gene ex datasets were retrieved from $E O, 1$ luding is array information of 27 OCCC san es and normal ovary samples. Gene expression and protes otein expression analyses based on public databases were $\mathrm{p}$ ormed to identify potential key genes correlated with OCQ and screen out DEGs. We identified 30 upregulated DEGs and 13 downregulated DEGs, which were mainly enriched in BPs in GO analysis, including tissue development, epithelium development, epithelial cell differentiation, tube development, organ development, and morphogenesis. MF analysis showed that the DEGs were significantly enriched in protein binding. For cell component, the DEGs were enriched in extracellular region and organelle.

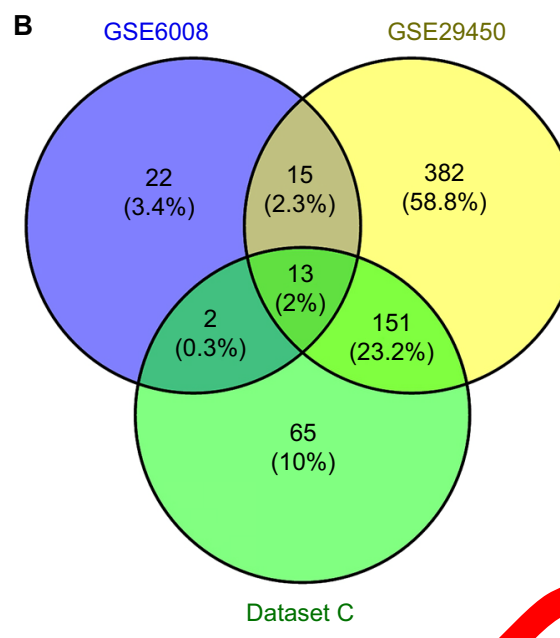

The results of KF path y analy showed that DEGs were mainly ed in ECM cor interaction pathway and TGF-bera signa o pathway.

By retwork an sis, six hub genes were identified ncluding WT1, SPPI, DCN, EPCAM, ALDH1A1, and $G A$ 16, two of $\mathrm{i}$ ich (SPP1, EPCAM) are upregulated in OCQ Four OC $C$ tumor tissue samples and four normal ovary tis noles were used to evaluate the expression ley the six hub genes by RT-PCR and Western blotting alysis. mRNA and protein levels of both SPP1 and EPCAM vere significantly upregulated in tumor than normal tissues hile no significant expression difference between groups was found for the other four downregulated genes .

EPCAM is a epithelial cell adhesion molecule (CAM) that does not belong to any of the four CAMs families (cadherins, selectins, integrins, and immunoglobulin-like CAMs) and discovered as one of the first cancer markers. ${ }^{12}$ It is a cell surface glycoprotein of $\sim 40 \mathrm{kDa}$ and is highly expressed in epithelial cancers. EPCAM may play key roles in the progression of ovarian cancer through promoting migration, proliferation, inhibiting cell apoptosis and adhesion, and disturbing cell cycle. It may be used as specific therapeutic targets in the treatment of ovarian cancer. ${ }^{13} E P C A M$ is suggested to be the DEGs between ovarian carcinomas and normal ovarian epithelium, indicating its involvement in the pathogenesis of ovarian cancer. ${ }^{14,15}$ Battista et al found that overexpression of EPCAM retains its significance independent of established prognostic factors for longer progression-free survival (PFS) (HR, 0.408; 95\% CI, 0.197-0.846; $P=0.003$ ) but not for PFS (HR, 0.666; 95\% CI, 0.366-1.212; $P=0.183) .{ }^{16}$ Another study indicated a significant association of EPCAM overexpres- 
Table 2 Significantly enriched GO terms and KEGG pathways of DEGs

\begin{tabular}{|c|c|c|c|c|}
\hline Classification & Term & Description & Counts & $P$-value \\
\hline BP term & GO:00096II & Response to wounding & 9 & $3.56 \mathrm{E}-02$ \\
\hline BP term & GO:007I37I & Cellular response to gonadotropin stimulus & 3 & $2.68 \mathrm{E}-02$ \\
\hline $\mathrm{BP}$ term & GO:0009888 & Tissue development & 17 & $1.03 \mathrm{E}-03$ \\
\hline BP term & GO:0060429 & Epithelium development & 12 & $3.60 \mathrm{E}-02$ \\
\hline BP term & GO:0030855 & Epithelial cell differentiation & 10 & I.32E-02 \\
\hline BP term & GO:0003006 & Developmental process involved in reproduction & 10 & $3.93 \mathrm{E}-03$ \\
\hline BP term & GO:0007548 & Sex differentiation & 7 & $5.55 \mathrm{E}-03$ \\
\hline BP term & GO:0045I37 & Development of primary sexual characteristics & 6 & $2.60 \mathrm{E}-02$ \\
\hline BP term & GO:0035295 & Tube development & 12 & $3.10 \mathrm{E}-03$ \\
\hline BP term & GO:00485I3 & Animal organ development & 19 & $3.33 \mathrm{E}-02$ \\
\hline BP term & GO:0009887 & Animal organ morphogenesis & 12 & $3.59 \mathrm{E}-03$ \\
\hline BP term & GO:0048645 & Animal organ formation & 5 & $2.98 \mathrm{E}-02$ \\
\hline BP term & GO:006/458 & Reproductive system development & 9 & I.09E-03 \\
\hline $\mathrm{BP}$ term & GO:0048608 & Reproductive structure development & 9 & OIE-03 \\
\hline BP term & GO:0008406 & Gonad development & 6 & 2. 02 \\
\hline BP term & GO:000I655 & Urogenital system development & 8 & $1.19,3$ \\
\hline BP term & GO:007200I & Renal system development & & $4.64 \quad 4$ \\
\hline BP term & GO:000I822 & Kidney development & & $2.7,-04$ \\
\hline BP term & GO:0090183 & Regulation of kidney development & 4 & $\nabla 9 \mathrm{E}-02$ \\
\hline BP term & GO:000I823 & Mesonephros development & & $8.4 \mathrm{IE}-03$ \\
\hline BP term & GO:0072006 & Nephron development & & $3.44 \mathrm{E}-02$ \\
\hline $\mathrm{BP}$ term & GO:0032835 & Glomerulus development & & $2.6 I \mathrm{IE}-02$ \\
\hline BP term & GO:00720I2 & Glomerulus vasculature development & 3 & 4.87E-02 \\
\hline BP term & GO:0072073 & Kidney epithelium development & 6 & I.6IE-03 \\
\hline BP term & GO:0072I63 & Mesonephric epithelium developmer & 5 & 6.90E-03 \\
\hline BP term & GO:0072I64 & Mesonephric tubule development & 5 & $6.90 \mathrm{E}-03$ \\
\hline BP term & GO:000I657 & Ureteric bud development & 5 & $6.56 \mathrm{E}-03$ \\
\hline BP term & GO:006I005 & Cell differentiation involved in $\mathrm{k}$ & 4 & I.7IE-02 \\
\hline BP term & GO:004666I & Male sex differentiation & 5 & $3.97 \mathrm{E}-03$ \\
\hline BP term & GO:0046546 & Development of prir & 5 & $1.68 \mathrm{E}-03$ \\
\hline BP term & GO:0008584 & Male gonad developm & 5 & I.6IE-03 \\
\hline BP term & GO:0090184 & Positive regulation of $k$ & 4 & $4.56 \mathrm{E}-03$ \\
\hline $\mathrm{CC}$ term & GO:0005576 & Extracelly on & 25 & I.50E-02 \\
\hline $\mathrm{CC}$ term & GO:004442I & Extrar alar reg h part & 25 & $4.27 \mathrm{E}-04$ \\
\hline $\mathrm{CC}$ term & GO:0043230 & melle & 21 & $2.45 \mathrm{E}-04$ \\
\hline $\mathrm{CC}$ term & GO:00056I5 & pace & 25 & \begin{tabular}{|l|}
$1.88 \mathrm{E}-04$ \\
\end{tabular} \\
\hline $\mathrm{CC}$ term & GO:I90356I & Extracel vesicle & 21 & $2.4 \mathrm{IE}-04$ \\
\hline $\mathrm{CC}$ term & GO:0070062 & Extracellula osome & 21 & $2.14 \mathrm{E}-04$ \\
\hline $\mathrm{CC}$ term & GO:0005796 & blgi lumen & 5 & $7.62 \mathrm{E}-03$ \\
\hline MF term & GO:0005 & Pr in binding & 39 & $8.79 \mathrm{E}-03$ \\
\hline KEGG_PATHWAY & $\mathrm{hsa} 04^{5}$ & ECM- eptor interaction & 3 & 0.0384428 \\
\hline KEGG_PATHWAY & hs? 50 & TGF-beta signaling pathway & 3 & 0.0409828 \\
\hline
\end{tabular}

Abbreviations: BP, biological process; $\mathrm{CG}^{\prime}$ ar compon tri DEGs, differentially expressed genes; ECM, extracellular matrix; GO, Gene Ontology; KEGG, Kyoto Encyclopedia of Genes and Ger

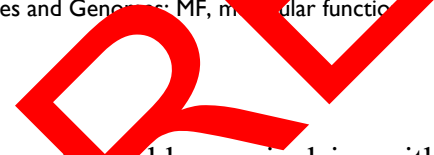

sion with a more is able survival in epithelial ovarian cancer patients. Serous cers showed a significant EPCAM overexpression compared with mucinous types in ovarian carcinoma. ${ }^{17}$ In addition, serum EPCAM level was found to be a diagnostic marker in epithelial ovarian cancer patients. ${ }^{18}$

Secreted phosphoprotein $1(S P P 1)$ is a secreted arginine glycine aspartic acid containing phosphorylated glycoprotein, with a molecular weight of about $325 \mathrm{kDa} .{ }^{19}$ The human SPP1 gene is located on chromosome 4 with seven exons and six introns. ${ }^{20}$ The expression of SPP1 is strongly related to tumor metastasis in gastric cancer and esophageal adenocarcinoma. ${ }^{21-23}$ Previous studies showed that SPP1 is highly expressed in many kinds of tumors, such as colon cancer, prostate cancer, lung cancer, and breast cancer. ${ }^{24-26}$ Modulation of vascular endothelial growth factor expression and regulation of extracellular matrix protein are the classic pathways in which SPP1 facilitates cancer progression. ${ }^{27,28}$ Recent study of SPP1 in epithelial ovarian cancer reveals that the expression of $S P P 1$ was higher in cancer tissues than in normal ovarian tissues. And it could be a useful biomarker 


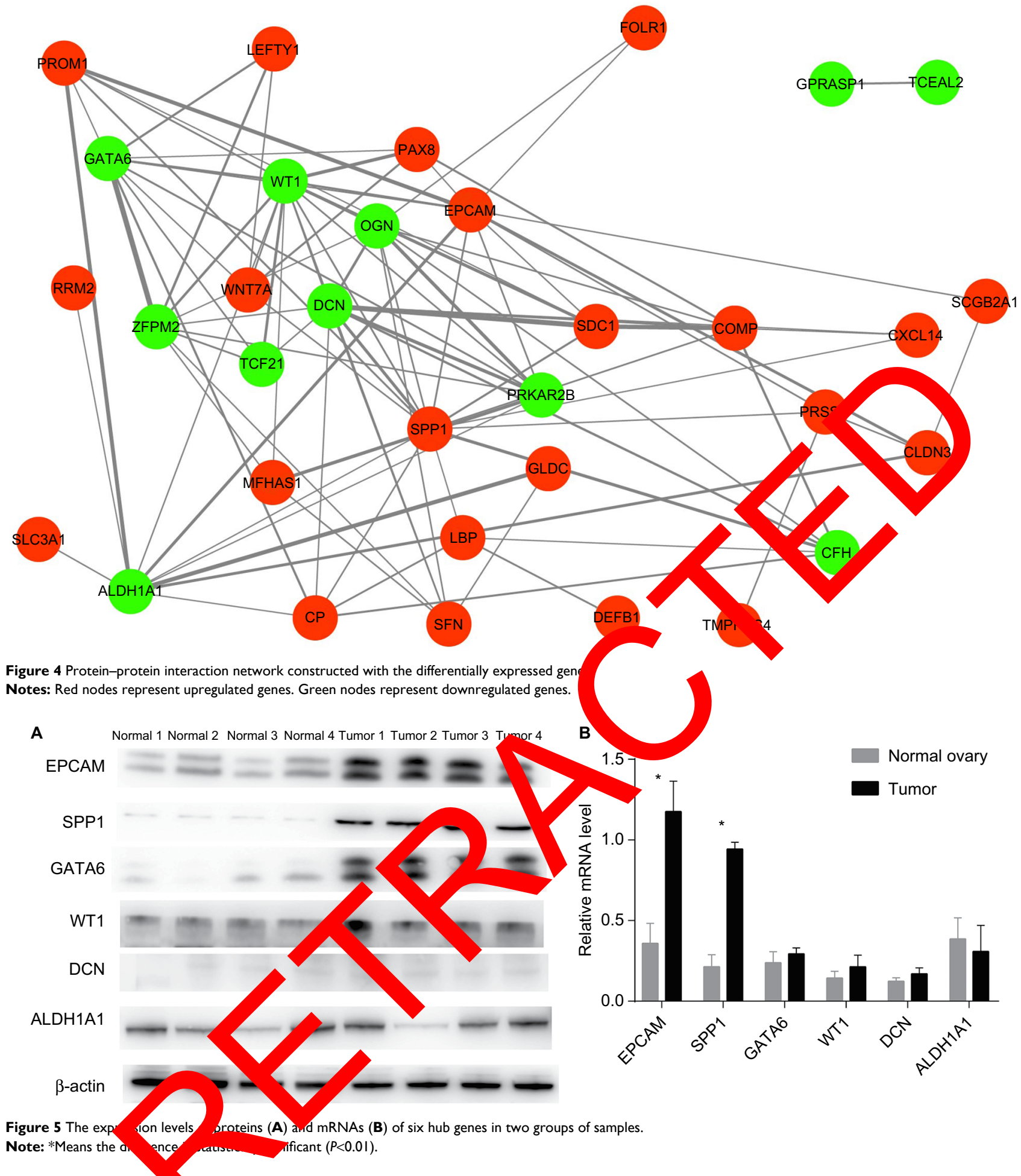

Table 3 Top six hub \& s with higher degree of connectivity

\begin{tabular}{|l|l|l|}
\hline Gene symbol & Gene description & Degree \\
\hline WTI & Wilms tumor I & 15 \\
\hline SPPI & Secreted phosphoprotein I & $\mathrm{I}$ \\
\hline DCN & Decorin & $\mathrm{I}$ \\
\hline EPCAM & Epithelial cell adhesion molecule & 12 \\
\hline ALDHIAI & $\begin{array}{l}\text { Aldehyde dehydrogenase I } \\
\text { family member AI }\end{array}$ & 10 \\
\hline GATA6 & GATA-binding protein 6 & 10 \\
\hline
\end{tabular}

in diagnosis of ovarian cancer with the diagnostic sensitivity and specificity of 0.66 (95\% CI, 0.51-0.78) and 0.88 (95\% CI, 0.78-0.93), respectively. ${ }^{29}$ Silencing SPP1 decreased the cell proliferation, migration, and invasion in vitro and prevented ovarian cancer growth in mice, during which the integrin $\beta 1 /$ FAK/AKT pathway was simultaneously inhibited. ${ }^{30}$ However, the expression and function of SPP1 in OCCC remain unclear. Therefore, $S P P 1$ may be a prognostic factor 
and potential therapeutic target for OCCC. However, larger multicenter analysis is still needed to confirm these results.

In our study, WT1, DCN, ALDH1A1, and GATA6 were downregulated in cancer compared to normal tissues by bioinformatic analysis. However, the RT-PCR and Western blotting analysis suggest otherwise. The role of these genes in OCCC is not clear. Experimental research of the biological functions of these genes in cancer cell lines is needed in the following study and can be illustrated in the future. Studies of large sample are required to evaluate the value of SPP1, EPCAM, and other genes in the prognostic evaluation and precise treatment of OCCC.

\section{Conclusion}

Our research identified six hub genes as potential key genes of OCCC by bioinformatic analysis. SPP1 and EPCAM are overexpressed in OCCC compared with normal ovary tissue. Experimental research is needed to reveal the biological functions of these genes in cancer cell lines. Clinical study of large sample is required to evaluate the value of SPP1 and EPCAM in the precision treatment and prognostic influence on OCCC.

\section{Acknowledgments}

This study was funded by the National Natural Science Foundation of China (81572576 [Shen Keng]) and the Chinese Academy of Medical Sciences (CAMS) Initiative for Innovative Medicine (CAMS-2018-12M-1-002 [Keng Shen]).

\section{Author contributions}

All authors contributed to data analysis, drafting and the article, gave final approval of the version to ba p and agree to be accountable for all aspects $0^{f}$

\section{Disclosure}

The authors report no conflicts of cerest in this wor

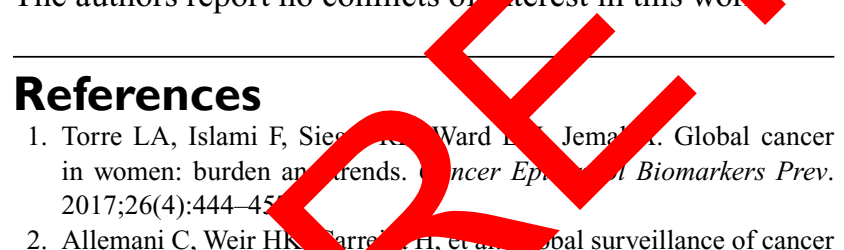

2. Allemani $\mathrm{C}$, Weir $\mathrm{Hh}$ arre $\mathrm{Hi}$, crm surveillance of cancer survival 1995-2009: anas of individual data for 25,676,887 patients from 279 population-based istries in 67 countries (CONCORD-2). Lancet. 2015;385(9972):977- 0 .

3. Schnack TH, Høgdall E, Nedergaard L, Høgdall C. Demographic clinical and prognostic factors of primary ovarian adenocarcinomas of serous and clear cell histology - a comparative study. Int J Gynecol Cancer. 2016;26(1):82-90.

4. Kim SI, Lee JW, Lee M, et al. Genomic landscape of ovarian clear cell carcinoma via whole exome sequencing. Gynecol Oncol.2018;148(2):375-382.

5. Pearce CL, Templeman C, Rossing MA, et al. Association between endometriosis and risk of histological subtypes of ovarian cancer: a pooled analysis of case-control studies. Lancet Oncol. 2012;13(4):385-394.
6. Mabuchi S, Kawase C, Altomare DA, et al. Vascular endothelial growth factor is a promising therapeutic target for the treatment of clear cell carcinoma of the ovary. Mol Cancer Ther. 2010;9(8):2411-2422.

7. Tan DS, Iravani M, McCluggage WG, et al. Genomic analysis reveals the molecular heterogeneity of ovarian clear cell carcinomas. Clin Cancer Res. 2011;17(6):1521-1534.

8. Gadducci A, Lanfredini N, Tana R. Novel insights on the malignant transformation of endometriosis into ovarian carcinoma. Gynecol Endocrinol. 2014;30(9):612-617.

9. Nagao-Kitamoto H, Shreiner AB, Gillilland MG, et al. Functional characterization of inflammatory bowel disease-associated gut dysbiosis in gnotobiotic mice. Cell Mol Gastroenterol Hepatol. 2016;2(4): 468-481.

10. Yamamoto S, Tsuda H, Takano M, Tamai S, Matsubara O. Loss of ARID1A protein expression occurs as an early event in ovarian clearcell carcinoma development and frequently coexists with PIK3CA mutations. Mod Pathol. 2012;25(4):615-624.

11. Cancer Genome Atlas Research Network. Integrated genomic analyses of ovarian carcinoma. Nature. 2011;474(7353):609-6

12. Trzpis M, McLaughlin PM, de Leij LM, Harm MC. E elial cell adhesion molecule: more than a carcinom larker and adh on molecule. Am J Pathol. 2007;171(2):386-395

13. Wang G, Zhang Z, Ren Y. TROP-1/Ep $\mathrm{AM}$ and 24 are $\mathrm{p}$ tial candidates for ovarian cancer $y^{\prime}$ apy. Int $J \mathrm{Cl}_{\mathrm{c}} \mathrm{\vee}_{\mathrm{x} p} \mathrm{hol}$. 2015;8(5):4705-4714.

14. Winge MC, Hoppe T, Berne B, et Filar in genotyne determines functional and molecular a ations an of pat with atopic dermatitis and ichthyosis garis. PLoS 201 or (12):e28254.

15. Thyssen JP, Kezic S. $C$ se. Cepidermal filc a reduction and their role in the pathogenesis of ato dermatitis. JAllergy Clin Immunol. 2014;134(4):792 709

16. Battista MJ vtarero C, Jakobi S, e . Overexpression of epithelial cell adhes a molecule protein is associated with favorable prognosis in an uns cted cohort of arian cancer patients. J Cancer Res Clin Oncol. 20 140(7):1097- 02.

17. Woopen $\mathrm{H}, \mathrm{K}$ ner $\mathrm{K}, \mathrm{R}$,er R, et al. Overexpression of the epithelial dhesion mon associated with a more favorable prognosis ad $\mathrm{res}_{\mathrm{l}}$ to platinum-based chemotherapy in ovarian cancer. $J$ nec sncom 14;25(3):221-228.

18. To Karabulut S, Serilmez M, Ciftci R, Duranyildiz D. Clinical sig icance of serum epithelial cell adhesion molecule (EPCAM) and vasc $r$ cell adhesion molecule-1 (VCAM-1) levels in patients with Thetial ovarian cancer. Tumour Biol. 2014;35(4):3095-3102.

19. Oldberg A, Franzén A, Heinegård D. Cloning and sequence analysis of rat bone sialoprotein (osteopontin) cDNA reveals an Arg-GlyAsp cell-binding sequence. Proc Natl Acad Sci U S A. 1986;83(23): 8819-8823.

20. Saitoh Y, Kuratsu J, Takeshima H, Yamamoto S, Ushio Y. Expression of osteopontin in human glioma. Its correlation with the malignancy. Lab Invest. 1995;72(1):55-63.

21. Lin J, Myers AL, Wang Z, et al. Osteopontin (OPN/SPP1) isoforms collectively enhance tumor cell invasion and dissemination in esophageal adenocarcinoma. Oncotarget. 2015;6(26):22239-22257.

22. Weng S, Zhou L, Han L, Yuan Y. Expression and purification of nontagged recombinant mouse SPP1 in E. coli and its biological significance. Bioengineered. 2014;5(6):405-408.

23. Zhuo C, Li X, Zhuang H, et al. Elevated THBS2, COL1A2, and SPP1 expression levels as predictors of gastric cancer prognosis. Cell Physiol Biochem. 2016;40(6):1316-1324.

24. Chuang CY, Chang H, Lin P, et al. Up-regulation of osteopontin expression by aryl hydrocarbon receptor via both ligand-dependent and ligand-independent pathways in lung cancer. Gene. 2012;492(1): 262-269.

25. Fedarko NS, Jain A, Karadag A, van Eman MR, Fisher LW. Elevated serum bone sialoprotein and osteopontin in colon, breast, prostate, and lung cancer. Clin Cancer Res. 2001;7(12):4060-4066.

26. Ue T, Yokozaki H, Kitadai Y, et al. Co-expression of osteopontin and CD44v9 in gastric cancer. Int J Cancer. 1998;79(2):127-132. 
27. Cui R, Takahashi F, Ohashi R, et al. Osteopontin is involved in the formation of malignant pleural effusion in lung cancer. Lung Cancer. 2009;63(3):368-374.

28. Hu Z, Lin D, Yuan J, et al. Overexpression of osteopontin is associated with more aggressive phenotypes in human non-small cell lung cancer. Clin Cancer Res. 2005;11(13):4646-4652.
29. Hu ZD, Wei TT, Yang M, et al. Diagnostic value of osteopontin in ovarian cancer: a meta-analysis and systematic review. PLoSOne. 2015;10(5):e0126444.

30. Zeng B, Zhou M, Wu H, Xiong Z. SPP1 promotes ovarian cancer progression via Integrin $\beta 1 /$ FAK/AKT signaling pathway. Onco Targets Ther. 2018;11:1333-1343.

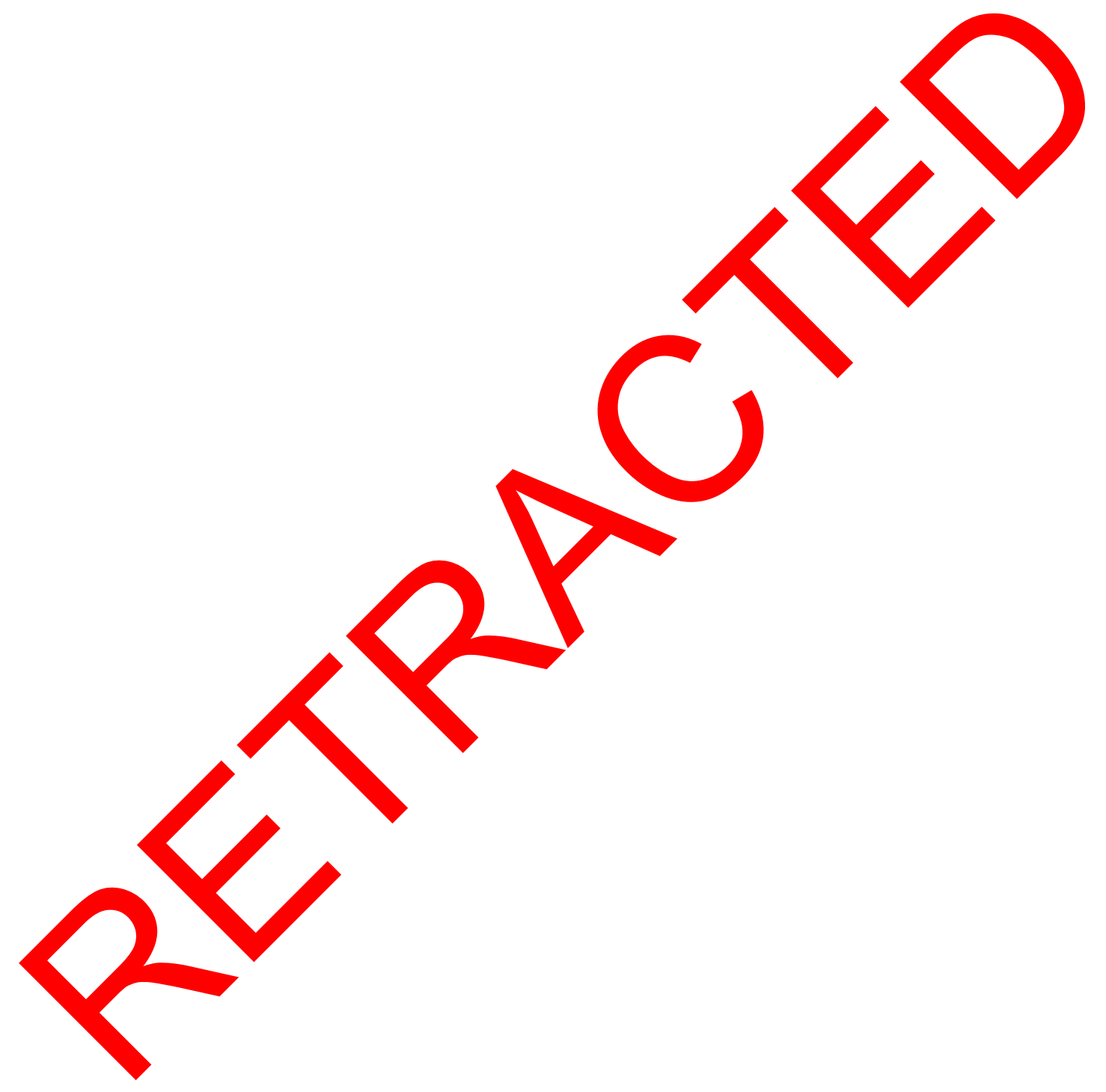




\section{Supplementary material}

Table SI Primer set for RT-PCR

\begin{tabular}{|c|c|c|}
\hline Gene & Forward primer & Reverse primer \\
\hline SPPI & 5'-CTCCATTGACTCGAACGACTC-3' & 5'-CAGGTCTGCGAAACTTCTTAGAT-3' \\
\hline WTI & 5'-CACAGCACAGGGTACGAGAG-3' & 5'-CAAGAGTCGGGGCTACTCCA-3' \\
\hline ALDHIAI & 5'-GCACGCCAGACTTACCTGTC-3' & 5'-CCTCCTCAGTTGCAGGATTAAAG-3 \\
\hline GATA6 & 5'-CTCAGTTCCTACGCTTCGCAT-3' & 5'-GTCGAGGTCAGTGAACAGCA-3' \\
\hline$D C N$ & 5'-ATGAAGGCCACTATCATCCTCC-3' & 5'-GTCGCGGTCATCAGGAACTT-3' \\
\hline EPCAM & 5'-AATCGTCAATGCCAGTGTACTT-3' & 5'-TCTCATCGCAGTCAGGATCATAA-3' \\
\hline GAPDH & 5'-GGAGCGAGATCCCTCCAAAAT-3' & 5'-GGCTGTTGTCATACTTCTCATGG-3' \\
\hline
\end{tabular}

Abbreviation: RT-PCR, real-time PCR.

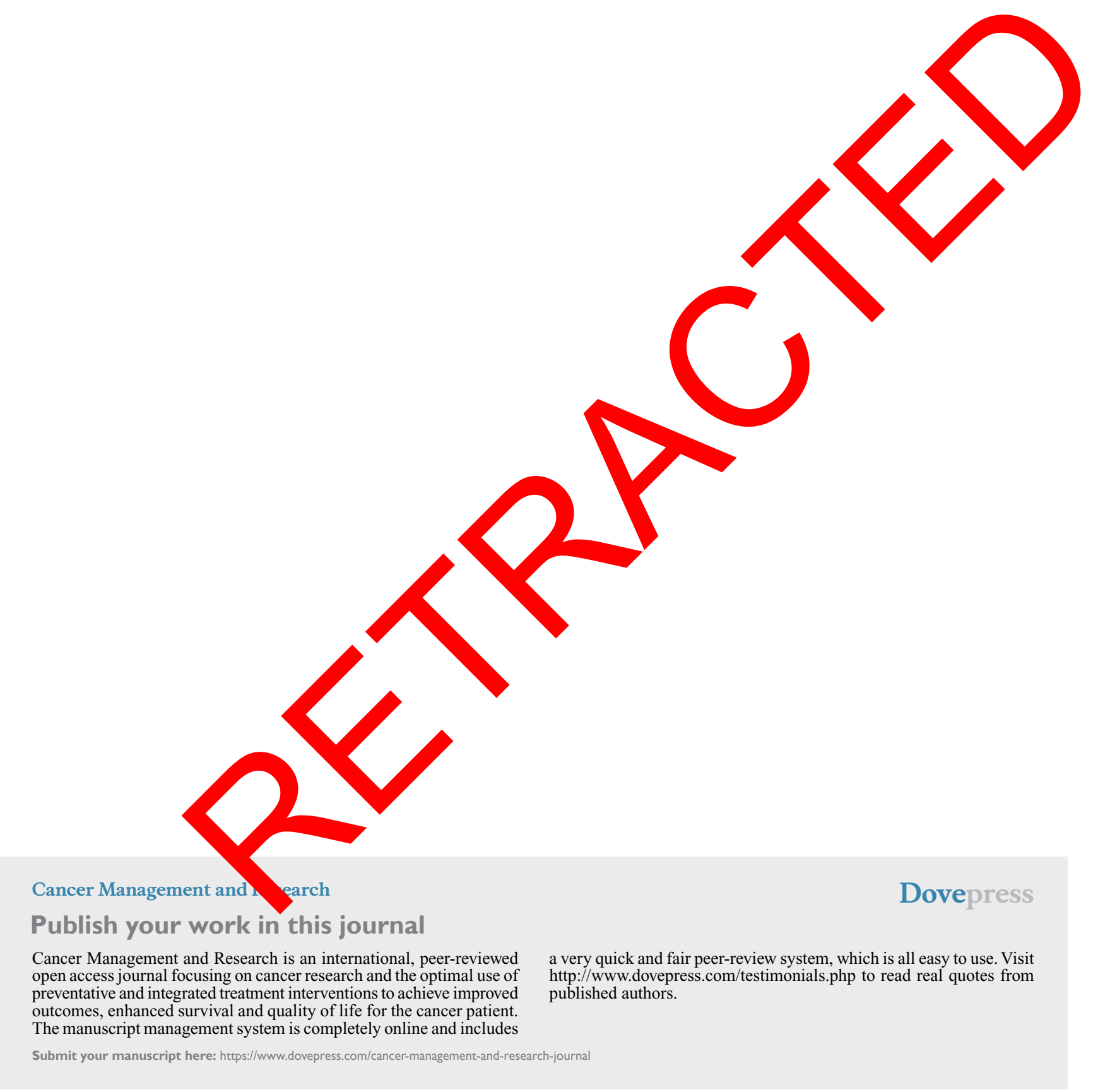

